\title{
Patients' self-evaluation of symptoms, signs and compliance to therapy for heart failure surveillance: A pilot study on identification of worsening heart failure
}

\author{
Vittorio Palmieri', Antonio Palermo² \\ 1 Heart Failure Outpatient Service, Cardiology Unit, "San Giuseppe Moscati” National Hospital, Avellino \\ 2 Cardiology Unit, "Sant'Anna e San Sebastiano" National Hospital, Caserta, Italy
}

\begin{abstract}
In patients with chronic heart failure (HF), it is unclear whether self-evaluation of HF-related symptoms and signs may contribute to the surveillance of clinical stability over time or the early identification of worsening HF. Therefore, HF-related symptoms and signs, and compliance to therapy were investigated by a self-administrated questionnaire in patients hospitalized due to worsening HF (cases) as well in those with stable HF (controls). The temporal interval of interest for the questionnaire focused on 2 to 7 days before the hospitalization or the control visit. A method called "classic" extrapolated a surveillance score by 4 questions revealing HF-related symptoms or signs (higher score indicative of more symptoms or sings reported). Alternatively, 3 additional questions enriched the "classic" questionnaire, including one on whether the health status was perceived as stable, improved or worsening. The score from "perceived health status" inquiry was used as "conditional factor" to computed the surveillance score as: conditional factor + [conditional factor X (the sum of the points yielded by the responses to each question on symptoms, sings and compliance to therapy)]. Among 51 patients hospitalized due to worsening $\mathrm{HF}$ and 104 with chronic HF (retrospective study), the area under the curve (receiver operating characteristic) discriminating worsening $\mathrm{HF}$ was 0.90 by the classic, and 0.96 by the conditional method (both $\mathrm{p}<0.001$ ),
\end{abstract}

Corresponding author: Vittorio Palmieri, Heart Failure Outpatient Service, Cardiology Unit, San Giuseppe Moscati" National Hospital, Contrada Amoretta, 83100 Avellino, Italy. E-mail: vpalmieri68@gmail.com

Key words: Heart failure; worsening; surveillance; questionnaire.

Contributions: VP, study design, patients' recruitment, data analysis, manuscript drafting and final approval of the manuscript; AP, study design reviewing, patients' recruitment, manuscript reviewing, final approval of the manuscript.

Conflict of interest: The authors declare no conflict of interest.

Received for publication: 24 February 2018

Accepted for publication: 22 June 2018

(C) Copyright V. Palmieri and A. Palermo, 2018

Tipografia PI-ME Editrice, Italy

Monaldi Archives for Chest Disease 2018; 88:919

doi: 10.4081/monaldi.2018.919

This article is distributed under the terms of the Creative Commons Attribution Noncommercial License (by-nc 4.0) which permits any noncommercial use, distribution, and reproduction in any medium, provided the original author(s) and source are credited. yielding error rates of 2 in 10 by the classic and 1 in 10 by the conditional method. In a pilot longitudinal study in 37 ambulatory HF patients from a different population source, predicting worsening HF by alternative questionnaires within 12 weeks from the visit yielded consistent results. In conclusion, patients' self-monitoring symptoms, signs and compliance by a standardized questionnaire using a conditional method for generating a relative score, may be a promising method for HF stability surveillance.

\section{Introduction}

Worsening heart failure (HF) is a challenging medical issue impacting prognosis, hospitalization length and outcome, and costs of the health system [1,2]. Early identification of HF-related symptoms and signs may trigger early treatment of worsening $\mathrm{HF}$ [3], reduce the number and the length of hospitalizations $[4,5]$, potentially contributing to outpatients intensive care programs in HF [6]. However, while HF is one of the chronic diseases associated with highest costs of management in primary care [7], intensive management in chronic HF may increase health management costs without relevant benefits [8]. Therefore, there is a need for research on surveillance of HF-stability impacting optimal disease management and costs. Sensitivity and specificity of early manifestation of worsening HF impact optimal disease management and costs as well [3-6].

Structured and relatively complex questionnaires are used with nurse-led support in HF to assess stability [9-12]. However, it remains unclear whether questionnaires on HF-related symptoms and signs [10,13-16] may be reliable in the setting of home environment and patients' self-administration without specific support. Therefore, the aim of our study was to investigate the probability of worsening HF assessed by scores derived from very short self-administrated questionnaires focused on HF-related symptoms, signs and compliance to therapy.

\section{Materials and Methods}

The study comprised a preliminary retrospective observation comparing self-reported HF-related symptoms, signs and compliance to therapy in patients hospitalized due to worsening $\mathrm{HF}$ and in stable ambulatory patients, and a prospective observation of incident worsening $\mathrm{HF}$ in ambulatory patients with chronic HF. The present spontaneous and unfunded observational study is based on clinical data from standard clinical practice. Patients gave informed consent to the anonymous utilization of their clinical data finalized to the amelioration of clinical practice and health assistance. The research respected the Code of Ethics of the World Medical Association (Declaration of Helsinki). 


\section{Retrospective study (testing sample)}

The study sample comprised patients hospitalized consecutively due to worsening HF in one of the following hospital Departments or Units: Emergency Department, Cardiology Unit, Internal and Emergency Medicine, Nephrology. Patients with worsening HF were defined as cases and were compared to ambulatory patients with stable $\mathrm{HF}$ (defined as controls). Exclusion criteria applied to worsening HF group of patients were: i) acute coronary syndrome defined as typical chest pain, ST segment deviation on standard electrocardiogram, and level of high sensitive troponin I 5 -fold higher than the upper normal limit declared by the laboratory; ii) acute inflammation defined as increased body temperature, increased high sensitive $\mathrm{C}$ reactive protein, increased white blood cells and response to antimicrobial therapy in 48-72 hours from admission; iii) pulmonary embolism; iv) end-stage cancer. HF was defined based on standard criteria, including symptoms and signs, structural cardiomyopathy assesses by echocardiography, increased B-type natriuretic peptide (BNP) above $250 \mathrm{pg} / \mathrm{ml}$ (normal values $<25$ ), or $250 \mathrm{pg} / \mathrm{ml}$ above the average of values while in stable chronic HF [17,18], and a remarkable reduction in symptoms and signs within $24 \mathrm{~h}$ from specific therapy with diuretics. Worsening HF was excluded in the Emergency Department or ambulatory HF unit in case of suspected worsening HF in the absence of B-type pulmonary lines by pulmonary echography, trans-mitral $\mathrm{E} / \mathrm{A}<0.7$, or $\mathrm{E} /$ lateral on pulsed tissue Doppler $<13$ and estimated systolic pulmonary pressure $<30 \mathrm{mmHg}$. Of the patients with worsening HF, 28\% were hospitalized in cardiology. Stable chronic HF was defined based on previous hospitalization due to HF with a clear response to diuretics, stable symptoms and signs and therapy (non-increment or reduction in diuretics), stable New York Heart Association functional classification. The recruitment was prolonged up to the target of 50 cases of worsening HF. In parallel, stable chronic HF patients were recruited from the outpatient unit. All patients received assistance to fill the Kansas City Cardiomyopathy Questionnaire (KCCQ) $[15,16]$.

\section{Simplified questionnaire for HF stability / instability}

A simplified questionnaire on symptoms and signs of HF was built in order to have patients able to self-evaluate HF stability without specific professional support, simulating home environment, according to previous experience on symptoms of acute and chronic volume overload $[13,14]$. In line with the validated simplified KCCQ [10], within the symptoms domain from the KCCQ [15,16], we selected 7 questions regarding symptoms \& signs with a time-interval of interest comprised between 2 and 7 days before the hospitalization or the ambulatory visit: question \#1-whether symptoms or signs were stable, worsening or improving (used only for the conditional method of computation of the overall score); question \#2- whether lower legs were swollen at wakeup in the morning; question \#3-whether patient had to sleep on a chair or sleep on 3 pillows when in bed due to shortness of breath; question \#4-whether shortness of breath or fatigue were limiting ordinary physical activity or self-care; question \#5-whether body weight increased 2 $\mathrm{kg}$ or more in 7 days before the access to the emergency department; question \#6-whther patient experienced syncope or near-syncope associated with palpitation as potential manifestation of tachyarrhythmias, a common trigger for worsening HF; question \#7-a question on regular assumption of specific medications as factor predicting worsening $\mathrm{HF}$ (Figure 1).

\section{Methods of computation of the global surveillance score-based symptoms, signs and compliance to therapy}

By a classic method (Figure 1, left side), the reported presence of symptoms or signs yielded 1 point per item, and final global surveillance score raged between 0 and 4 as it was the sum of the points for each item. By the conditional method, (Figure 1, right side), the question \#1 on perceived health status provided an score that "weighted" the overall surveillance score as reported in the Figure 1: points from items on symptoms and signs of possible worsening HF (6 in total related to acute or chronic volume overload $[4,14,17,18,19])$ yielded a partial total sum which was multiplied by the score from the "conditional factor" and added to it. Hence, the surveillance score from the conditional method was computed as: total conditional score $=$ conditional score (i.e. points from question \#1) + [conditional score X (sum of the scores for the symptoms and signs items)].

\section{Longitudinal pilot study (validation sample)}

A small longitudinal pilot study was conducted subsequently in ambulatory patients with stage C-D HF from a difference population source to test the performance of the different methods for HF stability surveillance in predicting worsening HF. Consecutive ambulatory patients clinically stable as defined earlier, were followed for 12 weeks from the ambulatory indexed visit. Patients filled-out the questionnaire weekly at home with results blinded to clinicians; patients underwent a control visit once a week including a clinical assessment and a pulmonary echography to define stable or worsening HF by standardized method $[3,17,20]$. Worsening HF was defined as clinical symptoms and signs of congestion concomitant to findings of wet lung by pulmonary echography, or pulmonary edema by chest X-ray or computed tomography scan, and symptoms and signs resolution within $72 \mathrm{~h}$ from the doubling of the usual oral dose of diuretic or intravenous treatment with diuretics.

\section{Echocardiographic methods}

Cardiac structure and functions were assessed by color-Doppler echocardiography by standardized methodology $[21,22]$.

\section{Statistical analyses}

Data in tables are mean \pm standard deviation, or percent. The Student's $t$-test for independent groups was used to evaluate differences in mean values for continuous variables. The Fisher's Exact test was used to compare differences in proportions between groups in 2 by 2 tables. Intraclass correlation coefficient was used to investigate the level of agreement between the classic and the conditional methods of computing the global score from the patients' questionnaire on HF-related symptoms and signs. The area under the curve of the receiver operating characteristic statistic, testing variability of true worsening $\mathrm{HF}$ (true positive, sensitivity) by false worsening HF (false positive or 1specificity) per incremental global score was used as measure of the strength of the odd of worsening HF by the incremental global score from patients' questionnaires. Differences between the areas under the curves obtained computing the global score of symptoms and signs by the classic and the conditional methods were tested by Z-statistic. 
Between 2 to 7 days before the access to the Emergency Department:

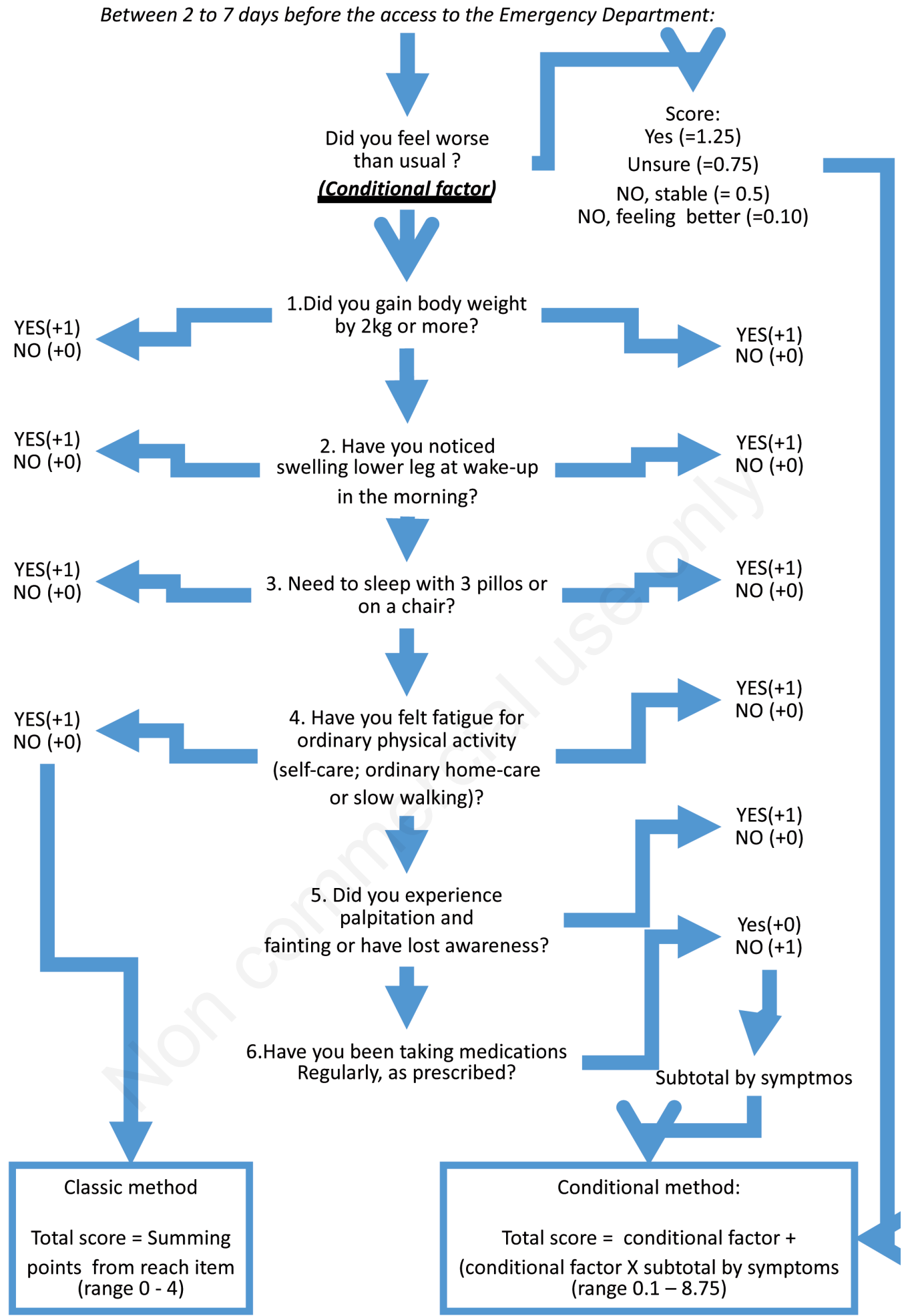

Figure 1. Questionnaire on self-assessment of HF-related symptoms and signs. Questions are reported in the central column. On the lefthand side, the method of computing a HF-stability score, so called classic, is illustrated. According to the formula reported at the bottom of the left column, classic score ranges between 0 and 4 , given the fact that each of the 4 questions yielded 1 point for the presence or 0 for the absence of the specific symptom or sign. The right-hand side column shows the 7 questions considered in the conditional method of computing the HF stability score. As it can be appreciated, the conditional method employed an initial question, the so called conditional factor, used to adjust the global score derived from symptoms, signs and compliance to therapy. The formula for computing the HF stability score by the conditional method is reported at the bottom of the right-had side column, with the global score ranging between 0.1 and 8.5 , in the specific case. 
With regards to the prospective pilot study, we tested the average symptoms and signs scores over the 12 weeks in patients who did not show worsening HF versus the scores in the week before the event (worsening HF). In all cases, the null hypothesis was rejected for $\mathrm{p}<0.05$.

\section{Results}

The retrospective study comprised 155 persons with worsening HF (cases) or with stable chronic HF (controls) in a 1:2 ratio (Table 1), in large majority of male gender in both groups. Those with chronic HF were older and showed lower blood pressure as compared to patients with worsening HF. The BNP was higher with acute HF and showed a wide intra-group standard deviation. The KCCQ was significantly lower in patients with worsening compared to those with chronic HF. Severely reduced left ventricular ejection fraction was common in both groups. On average, worsening HF was associated with a higher proportion of recurrent hospitalizations per year due to HF. Ischemic heart disease was common in both groups, whereas moderate-to severe kidney insufficiency or dialysis, moderate-to-severe mitral insufficiency and anemia were more prevalent among the worsening HF patients. History of atrial fibrillation was relatively common and equally prevalent in both groups; history of significant aortic valvular disease (i.e., valvular area $<1.5 \mathrm{~cm}^{2}$ and/or vena contract of the aortic insufficiency $>=5 \mathrm{~mm}$ ), of chronic obstructive pulmonary disease, of type II diabetes treated with insulin were relatively infrequent without differences between the two groups. The proportion of patients with automated implantable cardiac defibrillator was greater with chronic HF while the proportion of patients implanted with a cardiac resynchronization device was comparable between groups.

Table 2 summarizes the frequency of symptoms in the temporal interval of interest set between 2 and 7 days before the hospitalization, or the ambulatory visit. Among the patients with worsening HF, shortness of breath and/or fatigue for ordinary physical activity was reported fre-

Table 1. Clinical characteristics by heart failure type.

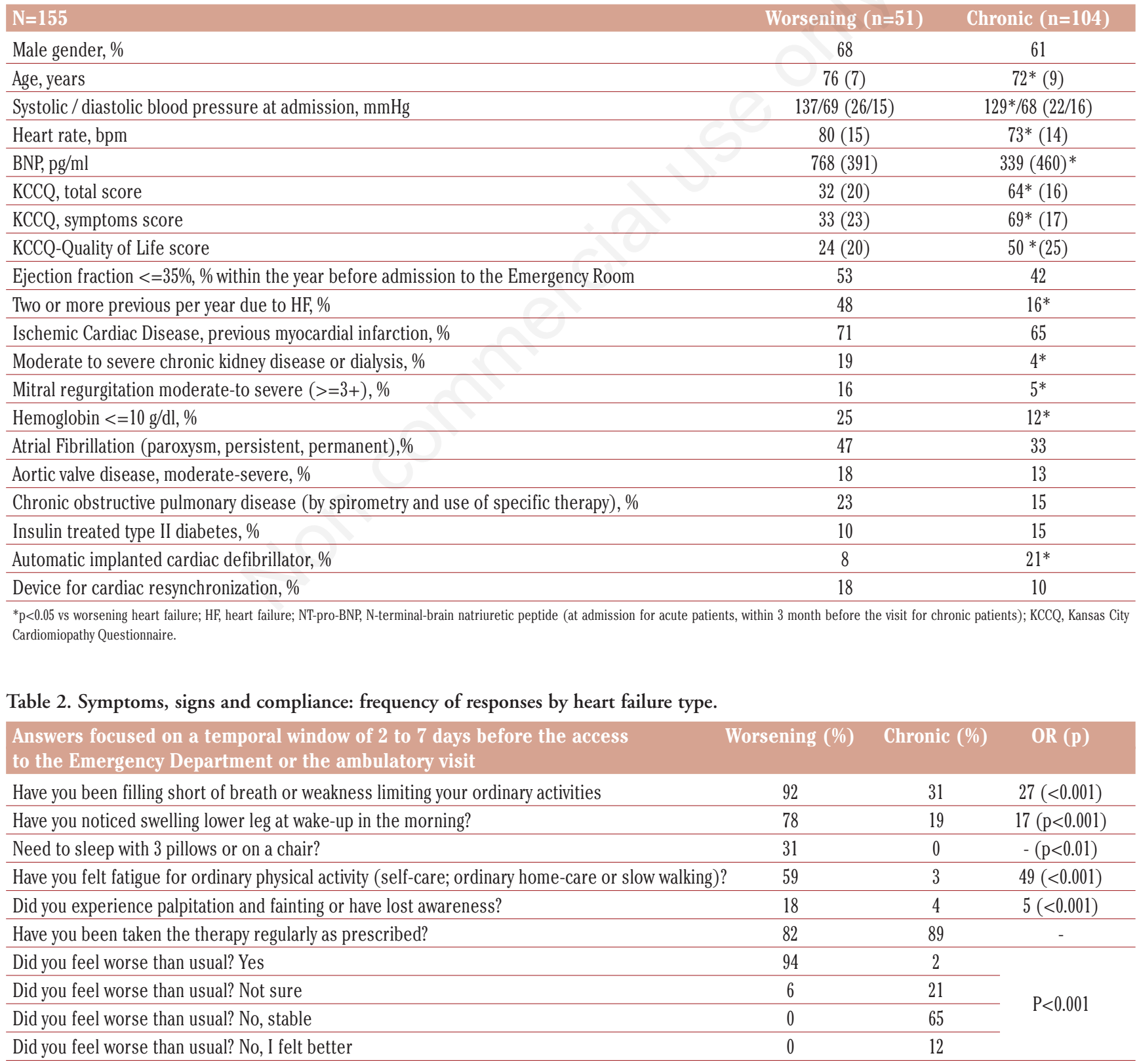


quently in both groups of patients. Swollen legs at wake-up in the morning was common before hospitalization for worsening HF, and significantly less frequent among patients with chronic HF. Orthopnea was reported only among patients who were hospitalized for worsening HF. Fatigue for ordinary physical activity and palpitaiont/fanting or loose of awareness were reported most frequently by patients who experienced worsening HF. A large majority of the patients self-reported to take regularly prescribed pills. A perception of worsening health condition was reported by a large majority of those who were hospitalized due to worsening $\mathrm{HF}$ and by a minority of those with chronic HF.

The global score assessed by the classic method showed a moderate agreement with that by the conditional method (intra-class correlation coefficient $0.70,95 \%$ confidence interval $0.14-0.87$ ).

With regards to worsening $v s$ chronic $\mathrm{HF}$, the area under the curve of the receiver operating characteristic was $0.91(\mathrm{p}<0.001)$ for the global score by the classic method (Figure 2A), and 0.98 ( $p<0.0001)$ for the conditional method (Figure 2B); overall, the classic method of assessment of the global score was associated with 2 errors of classification in 10 patients evaluated, whereas the conditional method was associated with 1 error of classification in 10 patients evaluated.

The small longitudinal pilot study comprised 37 patients with age $75 \pm 6$ years, $62 \%$ male gender, $62 \%$ with ischemic heart disease, $54 \%$ with left ventricular ejection fraction $<35 \%, 10 \%$ affected by insulintreated type II diabetes, $16 \%$ with severe chronic kidney disease (estimated creatinine clearance $<35 \mathrm{ml} / \mathrm{min}$ ) or on dialysis, all with at least one previous hospitalization for HF within one year from the index visit. Among those, $41 \%(n=15)$ experienced at least one episode of worsening HF within 3 months from the visit, requiring modification of the diuretic therapy or referral to nephrology in the case of patients on dialysis $(n=3)$; of those not on dialysis, all patients either doubled the usual oral dose of diuretics or received intravenous diuretic treatment in day-hospital (4 patients). By the area under the curve of the receiver operating characteristic statistic was 0.92 for the surveillance score by classic method and was 0.97 for that by the conditional method (both $\mathrm{p}<0.01)$.

\section{Discussion}

Our study evaluated whether patients' self-assessment of HF-related symptoms, signs and compliance to therapy in home-environment may be a reliable method for HF-stability surveillance. For the purpose, we employed a short set of questions similar in part to consolidated HF-related questionnaires [10] proven to be related to acute or chronic volume overload [14], and to the suspect or definite acute HF [17,18]. To minimize a potential tautology between the self-assessment HF instability to build the surveillance score and the definition of worsening $\mathrm{HF}$, both based on of symptoms and signs in large majority, in the retrospective study we defined the temporal interval of interest between 2 to 7 days before the hospitalization. We consistently found that shortness of breath and peripheral edema were the symptoms most frequently reported by patients hospitalized due to worsening HF [14]. The conditional method of evaluation of symptoms, signs and compliance showed the best discriminant performance, according to the receiver operating characteristic analyses. Such a method accounts for patients' perception of symptoms stability, episodes of palpitations or fainting or syncope, and information on the compliance to treatments, which allowed to refining discrimination between chronic and acute volume overload, and common triggers of worsening HF. While the validity of our results may reflect known findings such as the relationship between lower KCCQ in patients who experienced acute HF [11,12,23], our novel approach consisted in a very short list of questions for a very rapid assessment in home environment not requiring specific support for patients. Clearly, due to the nature of our study, use of the global HF-stability score to define a potential threshold for triggering interventions in definite or suspected worsening HF is beyond the scope of the current report, as the questionnaire needs to be tested in longitudinal long term studies. However, a pilot study in 37 patients showed initial encouraging results of a very low rate of false positive or false negative episodes of worsening HF, essentially characterized by worsening chronic volume

\section{Discriminating Worsening Heart Failure by Symptoms \& Signs Surveillance Score}

A

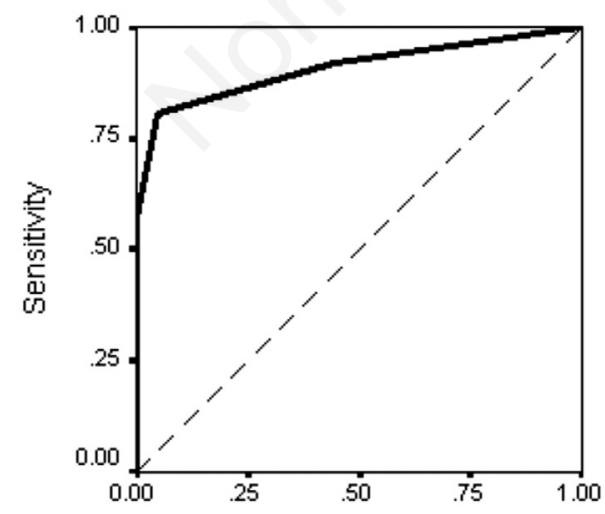

1 - Specificity
B

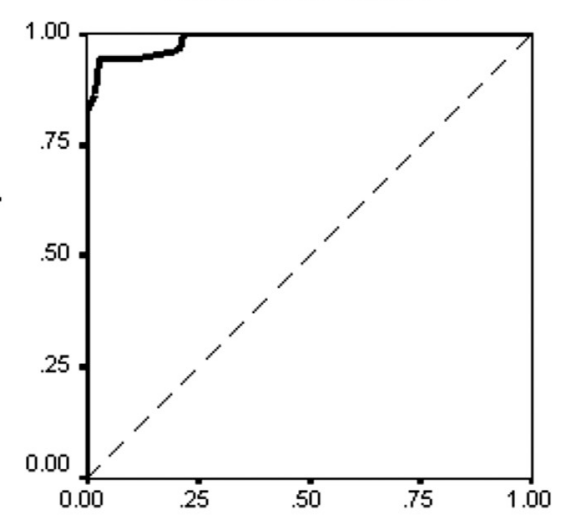

1 - Specificity

Figure 2. A) Trend of true positive (sensitivity, vertical axis) and false positive (1-specificity, horizontal axis) by the classic method for HF stability score. The area under the curve was $0.91(\mathrm{p}<0.01)$, with an expected accuracy (true positive + true negative over all participants) of 8 in 10 patients evaluated. B) Trend of true positive (sensitivity, vertical axis) and false positive (1-specificity, horizontal axis) by the conditional method for HF stability global score. The area under the curve was $0.98(\mathrm{p}<0.01)$, with an expected accuracy (true positive + true negative over all participants) of 9 in 10 patients evaluated. 
overload. The rate of events in the pilot prospective study was as high as $41 \%$ in 12 weeks, largely expected [24]. In a study comparing homebased versus clinic-based post-discharge management in HF [25], allcause hospitalization rate or death was as frequent as $88 \%$ in the whole cohort over 3.7 years of observation. At variance, in our pilot study, no patient was hospitalized, or died, over 3 months of observation, which predicts reduced healthcare management costs in the short term [26,27]. A previous meta-analysis in acute HF [17] found that the shortness of breath at rest was reported by the $45 \%$ of the patients admitted to the emergency room; nevertheless, shortness of breath or fatigue is common to clinical syndromes or diseases alternative to acute HF [19]. Hence, for rising the suspect of worsening HF, the use of a single item may be sensitive but not specific. Sensitivity with relatively low specificity may results in a significant rate of false positive events, and increased costs of management of those patients. On the other hand, in the setting of self-care in home environment, false negative cases with respect to potential worsening HF may be seen and a safety issue in HF surveillance programs, raising a relevant psychological burden for patients. The aggregation of a short set of items may increase the accuracy of the questionnaire, but specificity may increase while sensitivity may decrease. We experienced that with higher global score, the likelihood of true positive cases increased independently of the method used for computing the global surveillance score. So that, the substantial difference between the classic and the conditional method for assessing the global HF surveillance score may be appreciated for patients with a mild symptom/signs burden resulting in a relatively low global score by the classic method. Nevertheless, in the range of $0-2$ points by the classic method, the conditional method yielded a larger range of possible surveillance score, demonstrating de-correlation between the two methods and providing the base for a more accurate discrimination by the conditional approach. In fact, the area under the curve by the receiver operating characteristic was greater for the conditional method rather than for the classic method; extrapolating, the classic method may result in 2 errors (false positive or negative) in 10 patients while the conditional method in 1 error of classification every 10 patients evaluated. Of note, the conditional method employed a broader set of items correlated with worsening HF, such as compliance to prescribed medications [28], and also palpitations followed by near syncope or syncope, both correlated to triggers of HF [20].

The question on the perception of wellness, used as conditional factor, may fall into the domain of emotional factors [14], and be considered as a potential confounder. However, the answer to the question on perception of wellness yielded points that decreased the global score in all cases but when the patient perceived to feel worse (Figure 1), so that the conditional factor (perception of wellness) tend to reduce the impact of chronic symptoms associated with chronic stable volume overload. The pilot longitudinal study provided consistent results on the matter.

Our study has a number of limitations. We focused on worsening HF and excluded de novo cases. However, in the present context, we had chronic HF patients as controls, so that we aimed at a homogeneous experience on HF-related symptoms and signs between case and controls. The retrospective study should be considered as hypothesis generating. However, in preliminary data in a small group of patients from a difference population source, results tend to be consistent with regard to a small difference between the two methods, which may turn into a relevant difference in a large population.

\section{Conclusions}

A simple short questionnaire on HF-related symptoms, signs and compliance to therapy appears to be a promising tool for self-care sur- veillance to predict worsening $\mathrm{HF}$ and reduce the time-to-differential diagnosis and treatment. A conditional approach to estimate a global surveillance score for predicting worsening HF may refine the accuracy of a self-care surveillance. Large prospective studies are needed to validate our preliminary results.

\section{References}

1. Setoguchi S, Stevenson LW, Schneeweiss S. Repeated hospitalizations predict mortality in the community population with heart failure. Am Heart J 2007;154:260-266.

2. Alpert CM, Smith MA, Hummel SL, Hummel EK. Symptom burden in heart failure: assessment, impact on outcomes, and management. Heart Fail Rev 2017;22:25-39.

3. Mebazaa A, Yilmaz MB, Levy P et al. Recommendations on prehospital \& early hospital management of acute heart failure: a consensus paper from the Heart Failure Association of the European Society of Cardiology, the European Society of Emergency Medicine and the Society of Academic Emergency Medicine. Eur J Heart Fail 2015;17:544-558.

4. Matsue Y, Damman K, Voors AA et al. Time-to-Furosemide Treatment and Mortality in Patients Hospitalized With Acute Heart Failure. J Am Coll Cardiol 2017;69:3042-3051.

5. Inglis SC, Clark RA, McAlister FA et al. Structured telephone support or telemonitoring programmes for patients with chronic heart failure. Cochrane Database Syst Rev 2010;CD007228.

6. Jurgens CY, Lee CS, Reitano JM, Riegel B. Heart failure symptom monitoring and response training. Heart Lung 2013;42:273-280.

7. Carmona M, Garcia-Olmos LM, Garcia-Sagredo $P$ et al. Heart failure in primary care: co-morbidity and utilization of health care resources. Fam Pract 2013;30:520-524.

8. Boyne JJ, Van Asselt AD, Gorgels AP et al. Cost-effectiveness analysis of telemonitoring versus usual care in patients with heart failure: the TEHAF-study. J Telemed Telecare 2013;19:242-248.

9. Sawadogo K, Ambroise J, Vercauteren S et al. Interaction between the Kansas City Cardiomyopathy Questionnaire and the Pocock's clinical score in predicting heart failure outcomes. Qual Life Res 2016;25:1245-1255.

10. Spertus JA, Jones PG. Development and Validation of a Short Version of the Kansas City Cardiomyopathy Questionnaire. Circ Cardiovasc Qual Outcomes 2015;8:469-476.

11. Mishra RK, Yang W, Roy J et al. Kansas City Cardiomyopathy Questionnaire Score Is Associated With Incident Heart Failure Hospitalization in Patients With Chronic Kidney Disease Without Previously Diagnosed Heart Failure: Chronic Renal Insufficiency Cohort Study. Circ Heart Fail 2015;8:702-708.

12. Spertus J, Peterson E, Conard MW et al. Monitoring clinical changes in patients with heart failure: a comparison of methods. Am Heart J 2005;150:707-715.

13. Jurgens CY, Lee CS, Riegel B. Psychometric Analysis of the Heart Failure Somatic Perception Scale as a Measure of Patient Symptom Perception. J Cardiovasc Nurs 2017;32:140-147.

14. Jurgens CY, Moser DK, Armola R et al. Symptom clusters of heart failure. Res Nurs Health 2009;32:551-560.

15. Green CP, Porter CB, Bresnahan DR, Spertus JA. Development and evaluation of the Kansas City Cardiomyopathy Questionnaire: a new health status measure for heart failure. J Am Coll Cardiol 2000;35:1245-1255.

16. Kosiborod M, Soto GE, Jones PG et al. Identifying heart failure patients at high risk for near-term cardiovascular events with serial health status assessments. Circulation 2007;115:1975-1981. 
17. Martindale JL, Wakai A, Collins SP et al. Diagnosing Acute Heart Failure in the Emergency Department: A Systematic Review and Meta-analysis. Acad Emerg Med 2016;23:223-242.

18. Wang CS, FitzGerald JM, Schulzer M, Mak E, Ayas NT. Does this dyspneic patient in the emergency department have congestive heart failure? JAMA 2005;294:1944-1956.

19. Kato M, Stevenson LW, Palardy M et al. The worst symptom as defined by patients during heart failure hospitalization: implications for response to therapy. J Card Fail 2012;18:524-533.

20. Ponikowski P, Voors AA, Anker SD et al. 2016 ESC Guidelines for the diagnosis and treatment of acute and chronic heart failure: The Task Force for the diagnosis and treatment of acute and chronic heart failure of the European Society of Cardiology (ESC) Developed with the special contribution of the Heart Failure Association (HFA) of the ESC. Eur Heart J 2016;18:891-975.

21. Lang RM, Badano LP, Mor-Avi V et al. Recommendations for cardiac chamber quantification by echocardiography in adults: an update from the american society of echocardiography and the European association of cardiovascular imaging. J Am Soc Echocardiogr 2015;28:1-39.

22. Lancellotti P, Tribouilloy C, Hagendorff A et al. Recommendations for the echocardiographic assessment of native valvular regurgitation: an executive summary from the European Association of Cardiovascular Imaging. Eur Heart J Cardiovasc Imaging 2013;14:611-644.
23. Heidenreich PA, Spertus JA, Jones PG et al. Health status identifies heart failure outpatients at risk for hospitalization or death. J Am Coll Cardiol 2006;47:752-756.

24. Gundersen GH, Norekval TM, Haug HH et al. Adding point of care ultrasound to assess volume status in heart failure patients in a nurse-led outpatient clinic. A randomised study. Heart 2016;102:29-34.

25. Stewart S, Carrington MJ, Horowitz JD et al. Prolonged impact of home versus clinic-based management of chronic heart failure: extended follow-up of a pragmatic, multicentre randomized trial cohort. Int J Cardiol 2014;174:600-610.

26. Nuckols TK, Keeler E, Morton S et al. Economic Evaluation of Quality Improvement Interventions Designed to Prevent Hospital Readmission: A Systematic Review and Meta-analysis. JAMA Intern Med 2017;177:975-985.

27. Stewart S, Carrington MJ, Marwick TH et al. Impact of home versus clinic-based management of chronic heart failure: the WHICH? (Which Heart Failure Intervention Is Most Cost-Effective \& Consumer Friendly in Reducing Hospital Care) multicenter, randomized trial. J Am Coll Cardiol 2012;60:1239-1248.

28. Di Lenarda A, Scherillo M, Maggioni AP et al. Current presentation and management of heart failure in cardiology and internal medicine hospital units: a tale of two worlds - the TEMISTOCLE study. Am Heart J 2003;146:E12. 